\title{
La lecture à voix haute
}

Technique pour la compréhension active des textes

\section{Maria Victoria Reyzabal}

Traducteur : Claudine Adam

\section{(2) OpenEdition}

\section{Journals}

Édition électronique

URL : http://journals.openedition.org/ries/4271

DOI : 10.4000 /ries 4271

ISSN : 2261-4265

\section{Éditeur}

Centre international d'études pédagogiques

\section{Édition imprimée}

Date de publication : 1 juin 1994

Pagination : 83-86

ISSN : 1254-4590

\section{Référence électronique}

Maria Victoria Reyzabal, «La lecture à voix haute », Revue internationale d'éducation de Sèvres [En ligne], 02 | 1994, mis en ligne le 17 avril 2015, consulté le 19 avril 2019. URL : http://journals.openedition.org/ ries/4271 : DOl : 10.4000/ries.4271

Ce document a été généré automatiquement le 19 avril 2019

(c) Tous droits réservés 


\title{
La lecture à voix haute
}

\author{
Technique pour la compréhension active des textes
}

\author{
Maria Victoria Reyzabal
}

Traduction : Claudine Adam

1 La lecture à voix haute est écriture qui se voit et s'écoute et, comme telle, elle ajoute de nouvelles possibilités de compréhension à la lecture silencieuse; elle rend en outre possibles des interprétations personnelles plus riches, créatives, ludiques, etc.

\section{La didactique des codes pluriels}

2 L'enseignement et l'apprentissage des codes pluriels impliquent la connaissance des différentes manifestations du langage visuel, musical, gestuel, lorsqu'ils se combinent avec l'oral et l'écrit pour produire un texte nouveau, complexe, dont la compréhension, l'évaluation et la production exigent diverses compétences.

3 Le maniement des codes pluriels, et concrètement la lecture à voix haute, collaborent pour que les apprenants obtiennent de meilleurs résultats dans tout travail intellectuel et même manuel.

La réalisation d'une série d'activités immédiatement postérieures à l'audition [...] comme la représentation graphique et picturale d'épisodes déterminés, [...] le dialogue avec l'adulte, l'éclaircissement de la langue et des contenus, la discussion, la reprise favoriseront la découverte d'intérêts enfantins larges et autonomes et l'apparition d'intérêts nouveaux, susciteront l'enthousiasme pour le mot écrit, associant sa découverte à des moments d'intense gratification, ces activités peuvent, sans doute, assumer aussi une fonction de décharge émotive et agir positivement sur le développement intellectuel et l'élargissement des connaissances, l'enrichissement de l'imagination et du patrimoine linguistique, le perfectionnement de l'esprit critique et la formation d'une conscience morale claire 1 .

4 C'est pourquoi la lecture, en tant que voix qui s'écoute et se voit, enrichit la compétence communicative générale (compréhension et expression), car elle rend possibles :

- des relations cognitives à travers lesquelles la nouvelle information est intégrée aux expériences déjà accumulées ; 
- des relations sensorielles au moyen de manifestations exprimées par le texte écrit ;

- des relations logiques qui situent le temps et l'espace, la cause et l'effet, la partie et le tout, le genre et l'espèce ;

- des relations émotives qui engagent l'affectivité personnelle de celui qui lit et écoute ;

- des relations imaginatives qui facilitent la fabulation des modes personnels.

\section{La lecture à voix haute et l'écoute créative}

5 La lecture à voix haute considérée comme une activité créative et non comme une mécanique fastidieuse permet de créer des relations toujours nouvelles avec le monde environnant.

6 En ce qui concerne cette pratique de la lecture, il apparaît nécessaire que les élèves fassent la différence entre les utilisations propres du code oral, comme dans des situations de communication, et le passage à l'oral auquel se prête tout texte écrit. En apprenant à lire, on oublie trop souvent que certains phénomènes sonores sont difficiles à indiquer graphiquement (mélodie, rythme, pause...).

7 Une lecture à voix haute implique dans tous les cas une interprétation préalable même si elle est provisoire car il faut donner un sens approprié au texte au fur et à mesure qu'on le lit. La «musique » que l'on ajoute au texte écrit nécessite une interprétation, même si elle est hypothétique. Elle exige une anticipation de la part du lecteur pour intégrer le texte. Cette interprétation oriente, conditionne et peut même déterminer quelques aspects de la compréhension des auditeurs. Lire à haute voix exige de vocaliser significativement chaque mot, phrase et paragraphe dans son contexte discursif. Et c'est sans doute pour cette raison que certains textes ont besoin de ce type de lecture pour être appréciés à leur juste valeur. Tel est le cas, par exemple, des textes poétiques où l'on sera mieux en mesure d'apprécier les éléments stylistiques propres à ce genre particulier d'écriture. Une activité constructive à cet égard consiste à faire enregistrer par des élèves les mêmes lectures et de comparer ensuite ces enregistrements.

Le fait que des adultes lisent à voix haute à de jeunes enfants (voire à des " grands ", à des vieillards, à des malades...) peut être une expérience positive, complémentaire de la communication orale: en lisant, on oralise le langage utilisé par un autre et, par conséquent, on rend compte de son style.

Ainsi, la lecture favorise l'évaluation du texte écrit avec ses particularités propres et, comme nous venons de le signaler, favorise sa compréhension. À des fins didactiques, une telle lecture doit être préparée, il convient de créer un climat adéquat, de motiver les auditeurs (analyser des illustrations, commenter le sujet, mettre en relation l'histoire avec d'autres qui seraient connues...). Tout au long de cette lecture, on pourra formuler des hypothèses sur la trame, les personnages, le dénouement possible. À la fin de la lecture, chacun donnera son point de vue, fera part de ce qui a attiré son attention. Une scène pourra être dramatisée, des personnages réinventés, des épisodes ajoutés, la fin transformée.

Écouter de façon créative est possible, lorsque celui qui lit le fait bien.

Le lecteur créatif apporte à la littérature une imagination riche qui le rend capable de goûter pleinement ce qu'il ne peut pas comprendre en totalité ou qu'il n'avait ressenti qu'indirectement, surtout si celui qui l'introduit à la lecture à voix haute est quelqu'un qui en jouit et la pratique de façon créative. [...] L'auditeur participe 
au processus créatif en écoutant le lecteur recréer ce que l'auteur a imprimé. [...]

L'auteur et le lecteur mêlent leurs qualités artistiques².

11 La lecture à voix haute exige une interprétation qui aide les élèves à comprendre les structures morphosyntaxiques et textuelles, ce qui permet une interprétation plus rigoureuse et nuancée. Ceci est particulièrement net en ce qui concerne le théâtre lu et les récits dramatisés. C'est encore le cas pour la lecture à voix haute de mémoires, lettres, articles, lorsqu'on veut faire partager des informations, comparer des opinions ou ratifier des données car ce type de lecture facilite la réflexion en commun. Au cours du processus complexe de la lecture entrent en jeu non seulement les multiples compétences linguistiques que possède le sujet, mais encore ses connaissances para-linguistiques.

\section{La lecture et les différents types de textes}

12 Il est important d'obtenir des élèves qu'ils acquièrent l'habitude de la lecture sous ses différentes formes et fonctions et qu'elle soit faite à propos de textes d'auteurs, de styles, de genres différents. Chaque type de texte requiert une modalité de lecture.

13 Pour cela, il faut penser à diversifier les types de textes (narration, description, argumentation) et accéder à divers types de support du code écrit (livres, revues, journaux, prospectus, catalogues, cartes, mais aussi enregistrements sous des formes diverses).

On fuira les listes conventionnelles de livres ou de collections supposées valables en toutes circonstances. Ainsi, on pourra organiser un fichier avec les livres qui ont été jugés valables dans différentes situations et l'on retiendra les activités qui se sont révélées intéressantes avant ou après la lecture du point de vue de l'animation.

15 En utilisant la lecture à voix haute, on cherche à perfectionner l'élocution, à développer ou consolider une rapidité de lecture convenable, à stimuler l'intérêt pour la lecture mais aussi l'écoute et à préparer à d'autres pratiques orales ${ }^{3}$.

On peut affirmer que la lecture à voix haute est écriture qui se voit et s'écoute et comme telle, elle ajoute d'autres possibilités de compréhension à la lecture silencieuse. Elle permet de faciliter pleinement les interprétations personnelles, sur le mode actif, créatif, ludique. Elle encourage à faire d'autres lectures, voire à élaborer des productions personnelles.

\section{BIBLIOGRAPHIE}

FOUCAMBERT J. (1989), Cómo ser lector, Barcelona, Laia.

LOGAN L.M., LOGAN V.G. (1980), Estrategias para una enseñanza creativa, Vilassar de Mar, OikosTau.

NOBILE A. (1992), Literatura infantil y juvenil, Madrid, Morata-Ministerio de Educación y Ciencia.

REYZABAL M.V. (1993), La comunicación oral y su didáctica, Madrid, La Muralla. 
REYZABAL M.V., TENORIO P. (1992), El aprendizaje significativo de la literatura, Madrid, La Muralla. SIMON J. (1952), « Une batterie d'épreuves psychologiques pour la prédiction de la réussite en lecture », in Enfance, $n^{\circ} 5$.

\section{NOTES}

1. Nobile, A., 1992, 27-28.

2. Logan, L.M., Logan, V.G.: 1980, 267.

3. Reyzabal, M.V.: 1993, 195.

\section{RÉSUMÉS}

La lecture à voix haute apparaît comme une technique pour la compréhension active des textes, elle ajoute d'autres possibilités de compréhension à la lecture silencieuse et peut préparer à des pratiques créatives et des productions personnelles.

INDEX

Mots-clés : lecture, lecture à haute voix 